\title{
Phenylenediamine-based Carbon Nanodots Alleviate Acute Kidney Injury via Preferential Renal Accumulation and Antioxidant Capacity
}

Jie Gao ${ }^{1, \#, ~ Y a n f e n g ~ L i u ~}{ }^{2}$, , Bo Jiang ${ }^{1, \#, ~ W e n m i n ~ C a o ~}{ }^{1}$, Yanshen Kan ${ }^{1}$, Wei Chen ${ }^{1}$, Meng Ding', Guiyang Zhang ${ }^{2}$, Bowen Zhang ${ }^{2}$, Kai Xi $^{2,{ }^{*},}$ Xudong Jia ${ }^{2,}{ }^{*}$, Xiaozhi Zhao ${ }^{1,{ }^{*}}$, Hongqian $G u o^{l, *}$

1: Department of Urology, Nanjing Drum Tower Hospital, The Affiliated Hospital of Nanjing University Medical School, Institute of Urology Nanjing University, No. 321 Zhongshan Rd, Nanjing, Jiangsu, China.

2: School of Chemistry \& Chemical Engineering, Nanjing University, No. 163

Xianlin Rd, Nanjing, Jiangsu, China.

\#These authors contributed equally to this work.

*Corresponding authors:

Hongqian Guo: e-mail: dr.ghq@nju.edu.cn Telephone number: 862583106666; Fax:

862568182863; Address: No. 321 Zhongshan Road, Nanjing, Jiangsu Province,

China. 210008.

Xiaozhi Zhao: zhaoxz@nju.edu.cn_Xudong Jia: jiaxd@nju.edu.cn

Kai Xi: xikai@nju.edu.cn 




Figure S1. A schematic illustration for the synthesis of PDA-CNDs, 2,4-DAT-CNDs, 2,6-DAT-CNDs and MPDSA-CNDs.
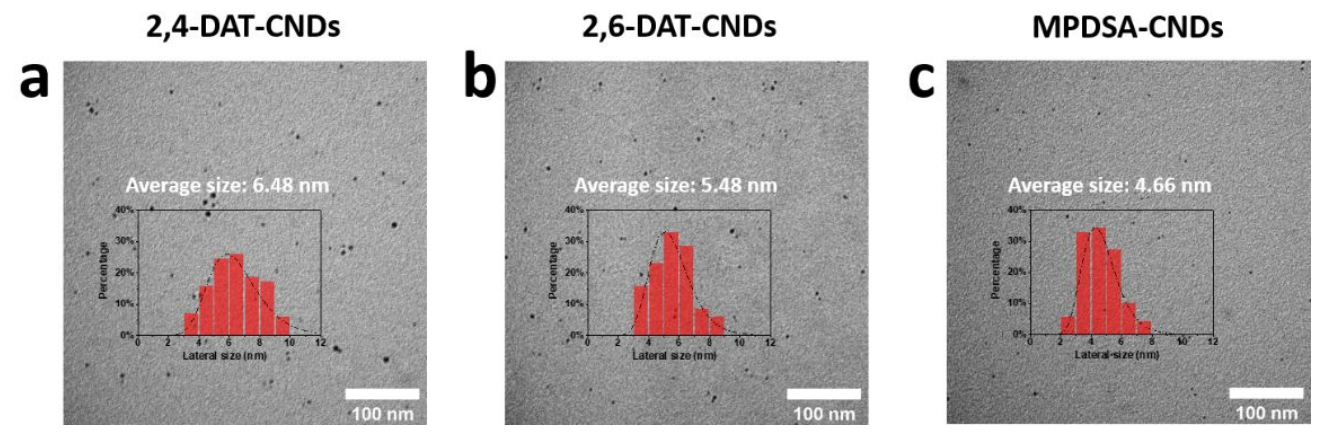

Figure S2. TEM image and lateral size distribution (inset) of 2,4-DAT-CNDs (a), 2,6DAT-CNDs (b) and MPDSA-CNDs (c).

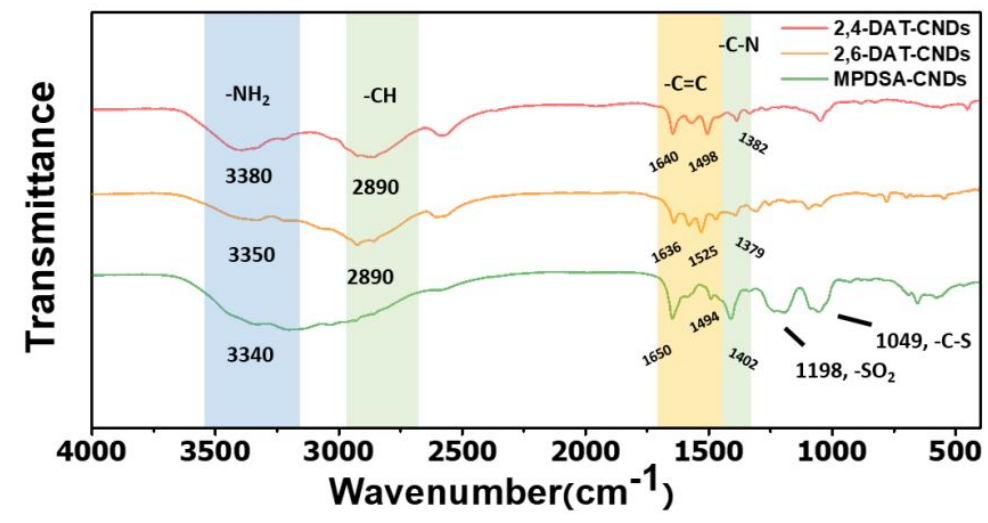

Figure S3. FT-IR transmittance spectra for 2,4-DAT-CNDs, 2,6-DAT-CNDs and MPDSA-CNDs. 
a

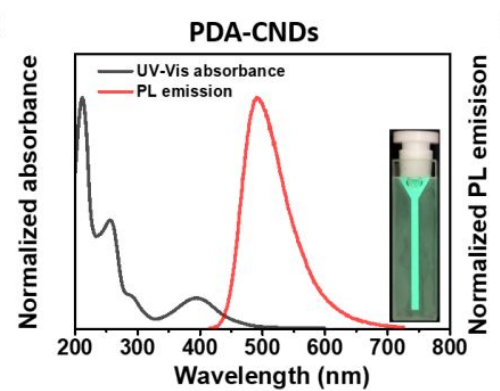

b

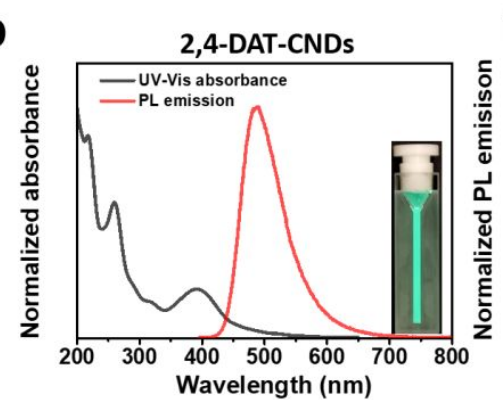

C



d



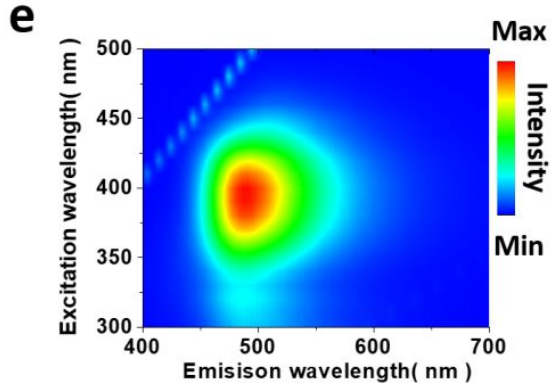
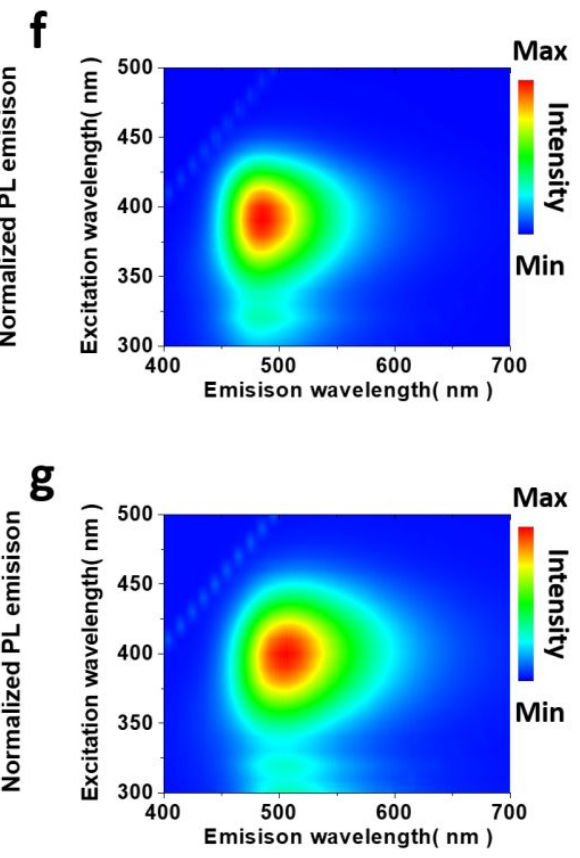

h



Figure S4. UV-Vis absorbance and PL emission features for PDA-CNDs, 2,4-DATCNDs, 2,6-DAT-CNDs and MPDSA CNDs. (a-d) Normalized UV-Vis absorbance and PL emission (under $365 \mathrm{~nm}$ excitation) spectra for PDA-CNDs (a), 2,4-DAT-CNDs (b), 2,6-DAT-CNDs (c) and MPDSA-CNDs (d) (inset: photograph of CNDs aqueous solution under 365-nm UV irradiation); (e-h) 3D-PL contour of PDA-CNDs (e), 2,4DAT-CNDs (f), 2,6-DAT-CNDs (g) and MPDSA-CNDs (h). 
a



b

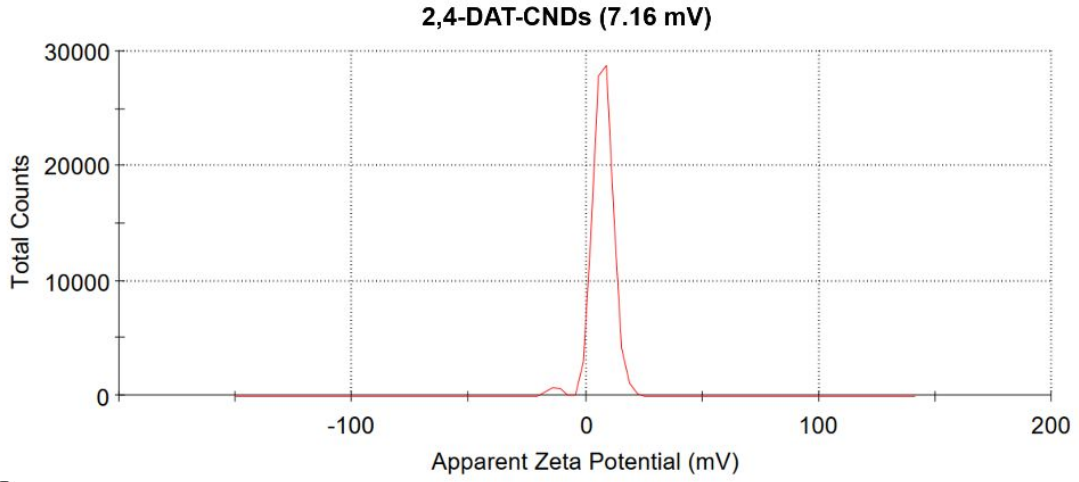

C

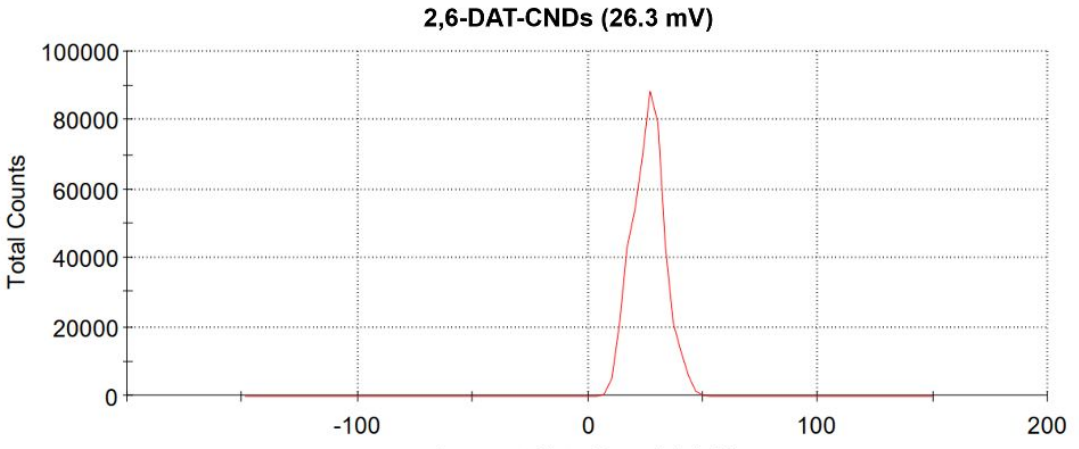

d

Apparent Zeta Potential (mV)

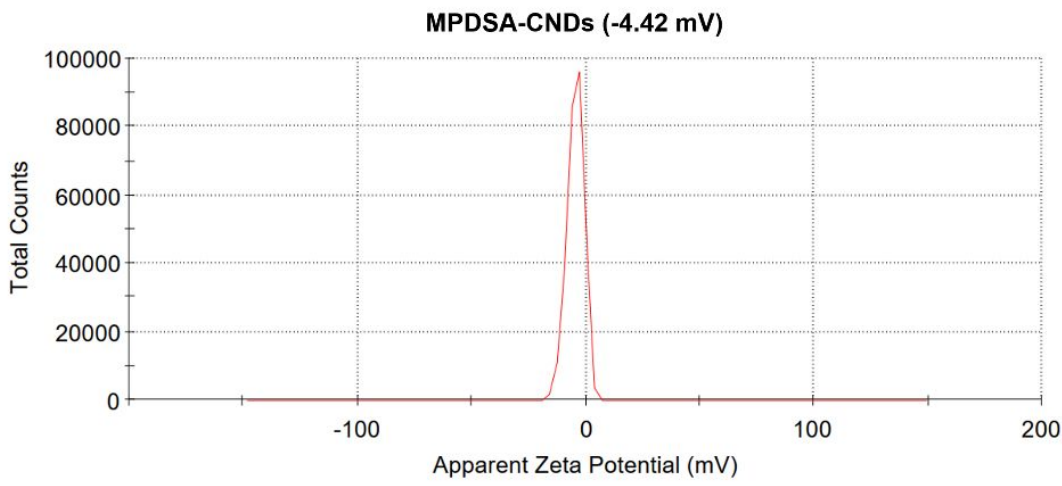

Figure S5. Apparent Zeta potential of the four CNDs. (a) PDA-CNDs, (b) 2,4-DATCNDs, (c) 2,6-DAT-CNDs and (d) MPDSA-CNDs. 
a

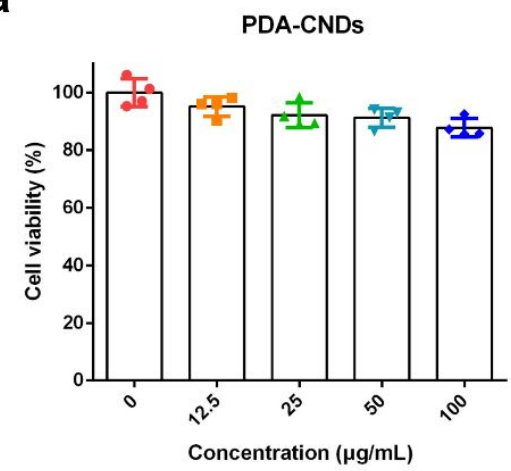

C

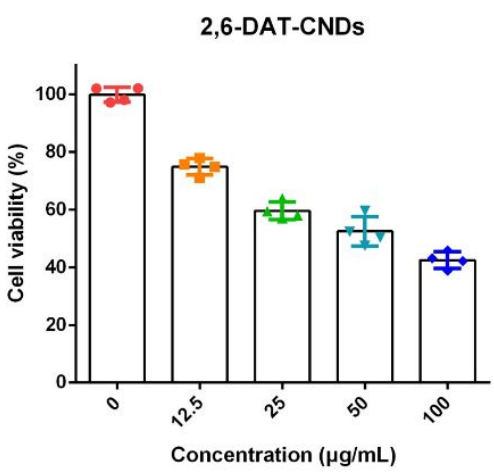

b



d

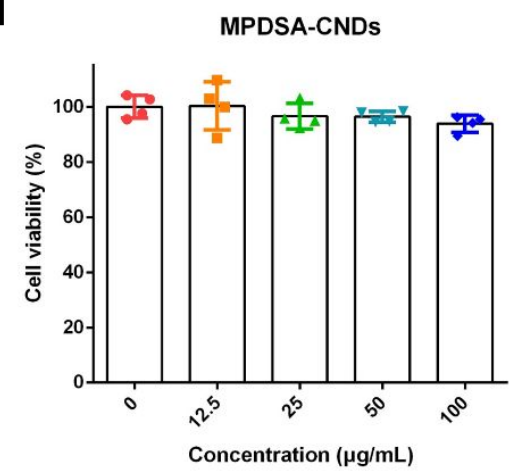

Figure S6. Cellular toxicity of different CNDs to HK2 cells. (a) PDA-CNDs, (b) 2,4DAT-CNDs, (c) 2,6-DAT-CNDs and (d) MPDSA-CNDs. Data are mean \pm SD from four independent replicates.
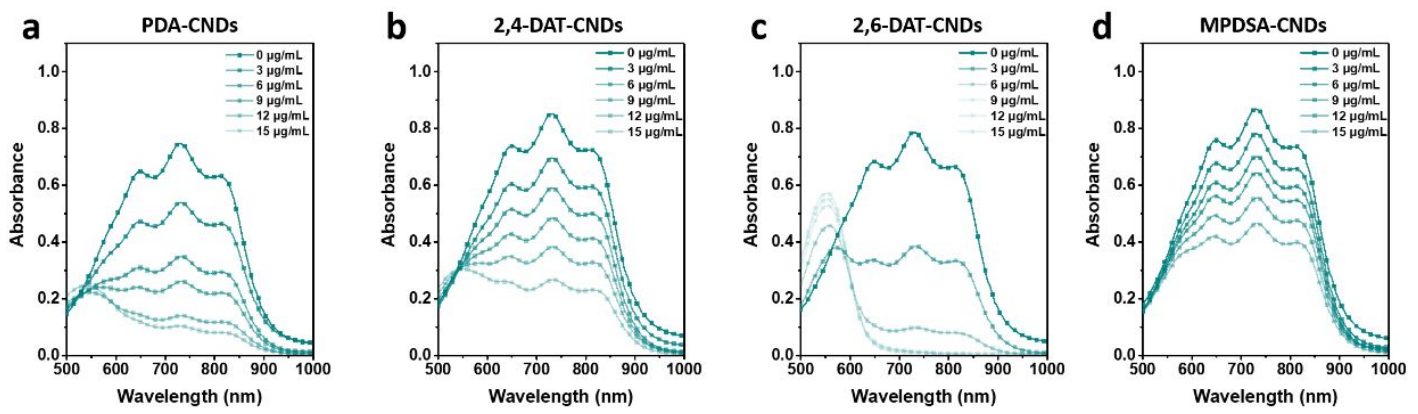

Figure S7. The absorption spectra of ABTS radical with CNDs of different concentrations. ABTS radical absorbance with (a) PDA-CNDs, (b) 2,4-DAT-CNDs, (c) 2,6-DAT-CNDs and (d) MPDSA-CNDs. 

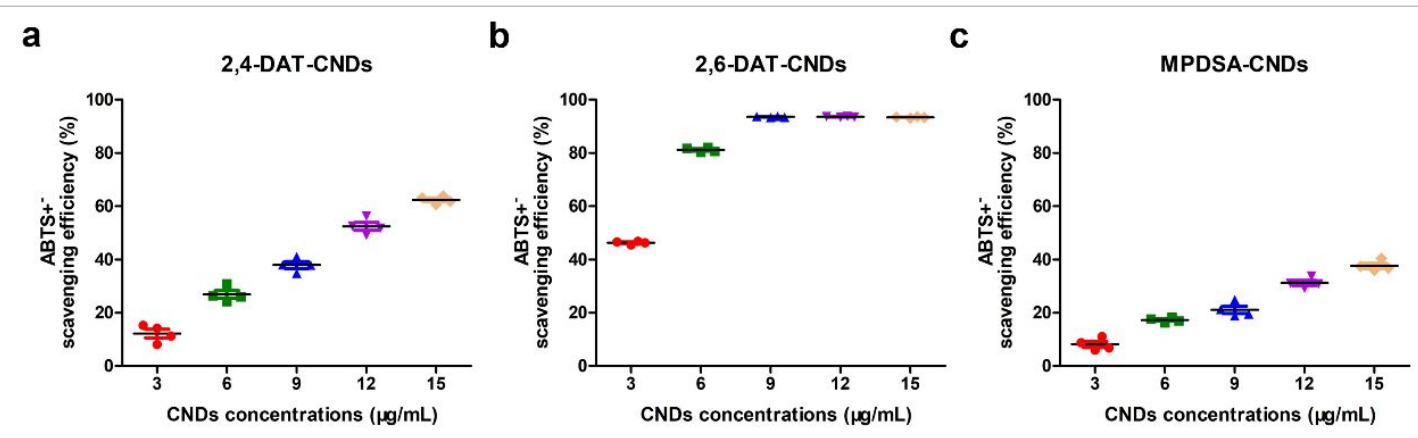

Figure S8. Scavenging efficiency of ABTS radical with CNDs of different concentrations. (a) 2,4-DAT-CNDs, (b) 2,6-DAT-CNDs and (c) MPDSA-CNDs. Data are mean $\pm \mathrm{SD}$ from four independent replicates.

a

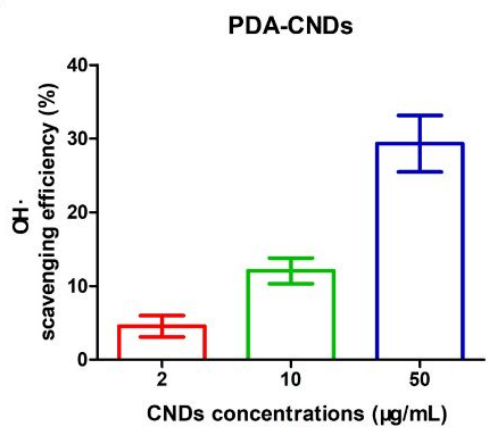

C

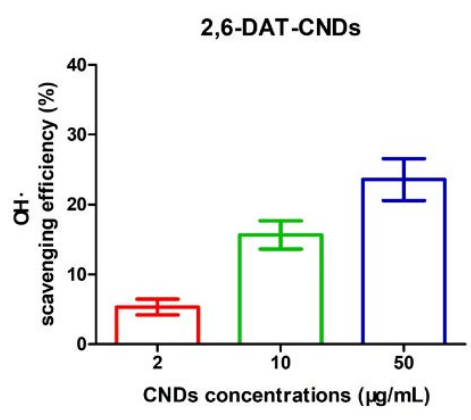

b

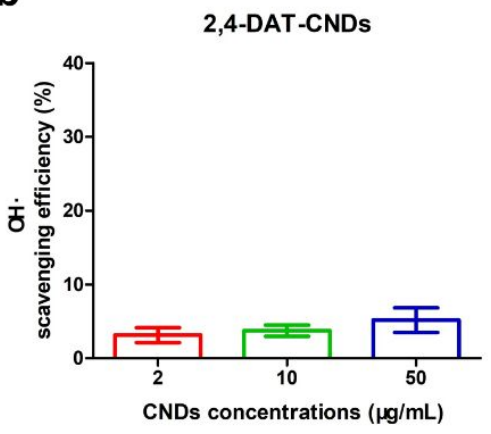

d

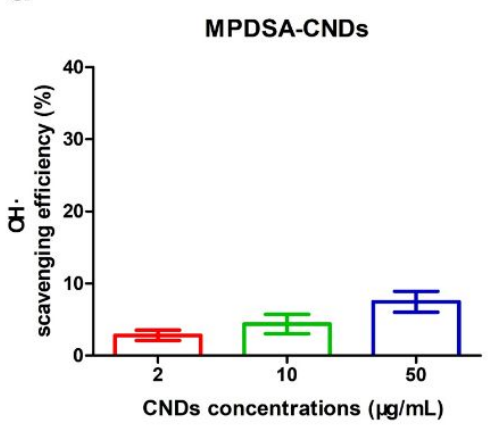

Figure S9. Scavenging efficiency of $\mathrm{OH} \cdot$ with $\mathrm{CNDs}$ of different concentrations. (a) PDA-CNDs, (b) 2,4-DAT-CNDs, (c) 2,6-DAT-CNDs and (d) MPDSA-CNDs. Data are mean $\pm \mathrm{SD}$ from three independent replicates. 

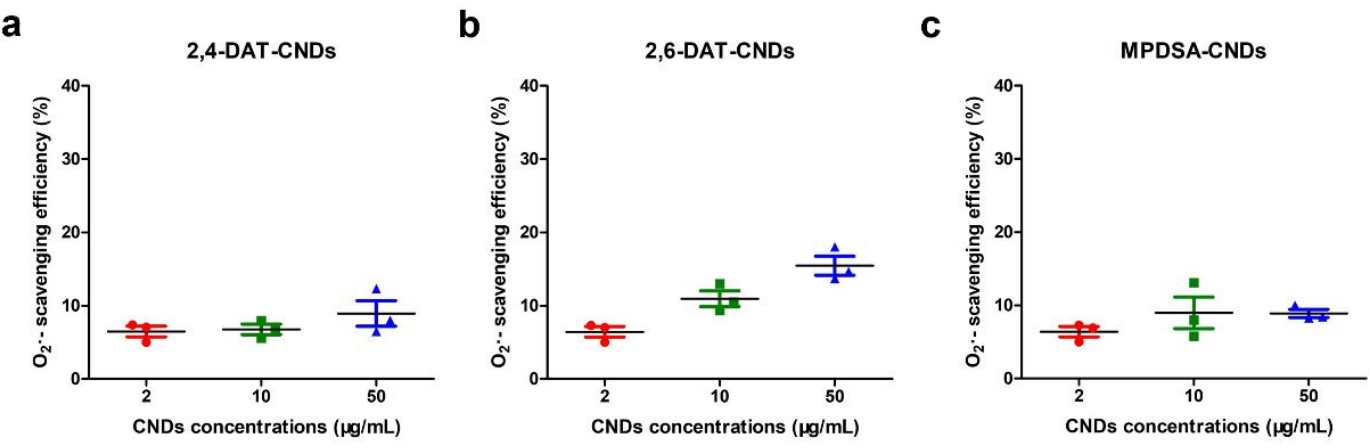

Figure S10. Scavenging efficiency of $\cdot \mathrm{O}_{2}^{-}$with CNDs of different concentrations. (a) 2,4-DAT-CNDs, (b) 2,6-DAT-CNDs and (c) MPDSA-CNDs. Data are mean \pm SD from three independent replicates.

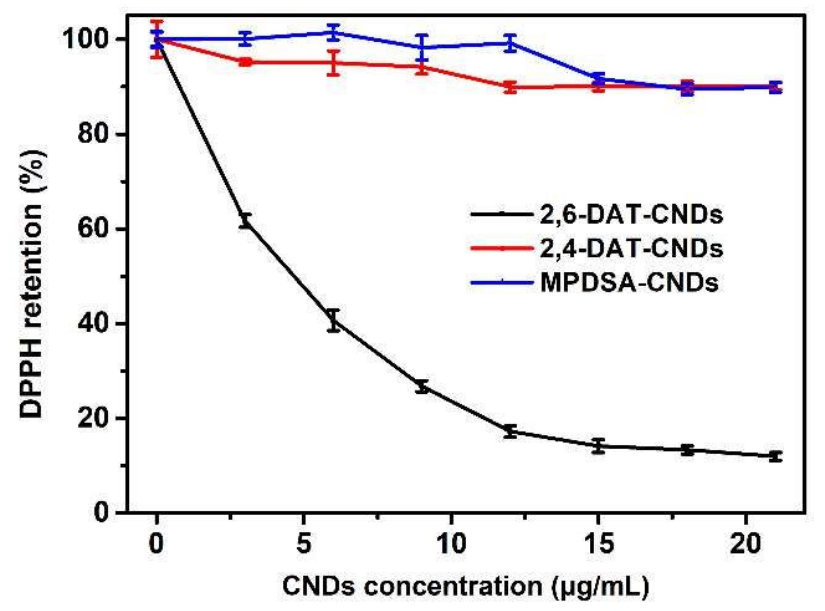

Figure S11. Scavenging efficiency of DPPH radical with CNDs of different concentrations. Data are mean \pm SD from five independent replicates. 

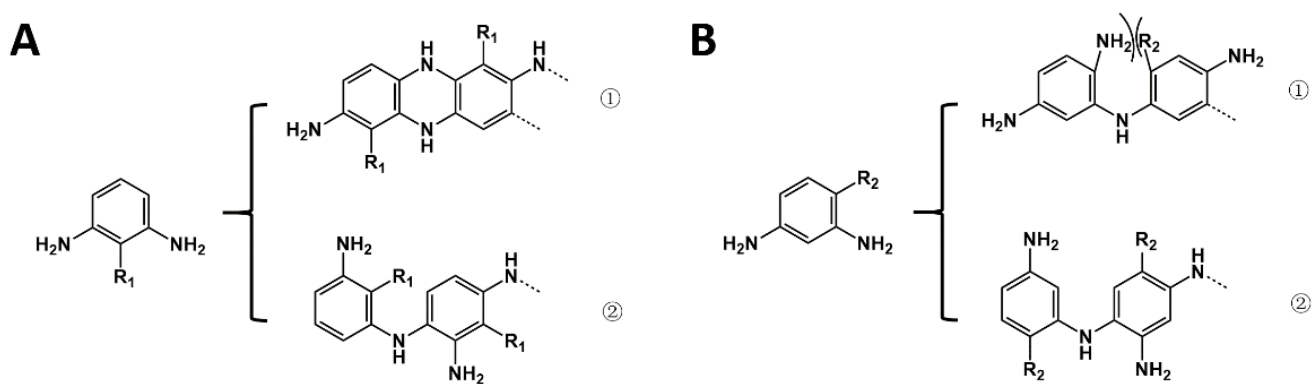

Figure S12. Detailed structure of each CNDs. As showed above, for PDA and 2,6-DAT (part A, R1 = H or $\mathrm{CH}_{3}$ ), both the structure (1) and structure (2) may occur during the formation of CNDs, meanwhile for 2,4-DAT and MPDSA (part B, R2 $=\mathrm{CH}_{3}$ or $\mathrm{SO}_{3}$ ), only the structure (2) were formed due to the steric hindrance. We speculated that introducing structure (1) might have provided advantages for the enhancement of ROS scavenging capacity for 2,6-DAT-CNDs and PDA-CNDs.
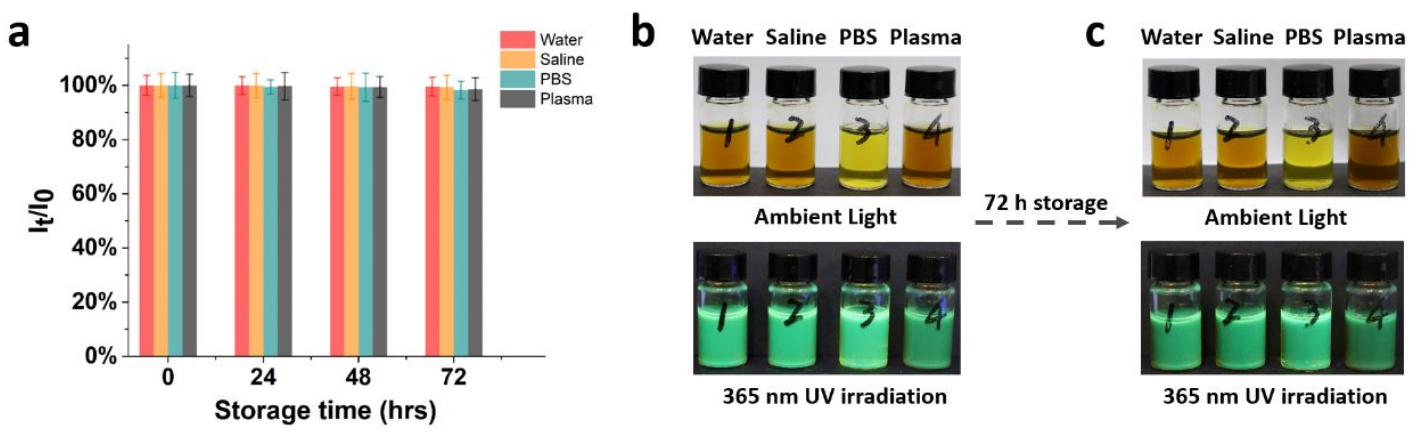

Figure S13. The dispersion stability of PDA-CNDs $(500 \mu \mathrm{g} / \mathrm{mL})$ in different media (deionized water, normal saline, PBS buffer and mice plasma) under $4^{\circ} \mathrm{C}$. (a) Ratio of final fluorescence intensity $\left(\mathrm{I}_{\mathrm{t}}\right)$ and initial fluorescence intensity $\left(\mathrm{I}_{0}\right)$ of PDA-CNDs solutions before and after storage, plotted against storage time. (b) Photographs of PDA-CNDs solutions before storage and under ambient light and $365 \mathrm{~nm} \mathrm{UV}$ irradiation. (c) Photographs of PDA-CNDs solutions before $72 \mathrm{~h}$ storage and under ambient light and $365 \mathrm{~nm}$ UV irradiation. 


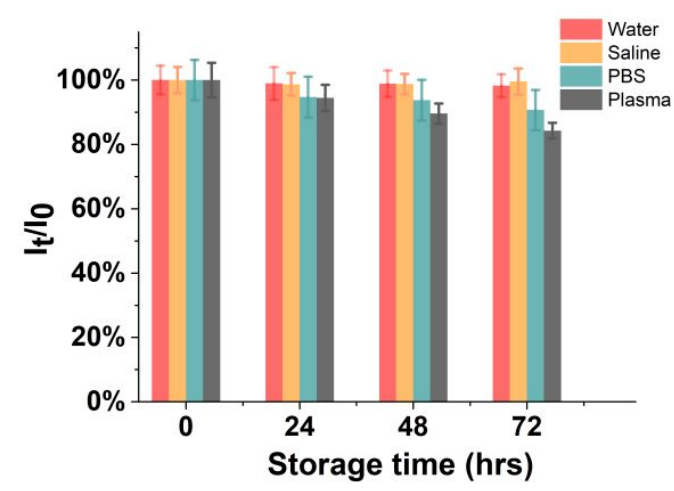

Figure S14. The dispersion stability of PDA-CNDs $(500 \mu \mathrm{g} / \mathrm{mL})$ in different media (deionized water, normal saline, PBS buffer and mice plasma) under $37^{\circ} \mathrm{C}$. Ratio of final fluorescence intensity $\left(\mathrm{I}_{\mathrm{t}}\right)$ and initial fluorescence intensity $\left(\mathrm{I}_{0}\right)$ of PDA-CNDs solutions before and after storage, plotted against storage time.

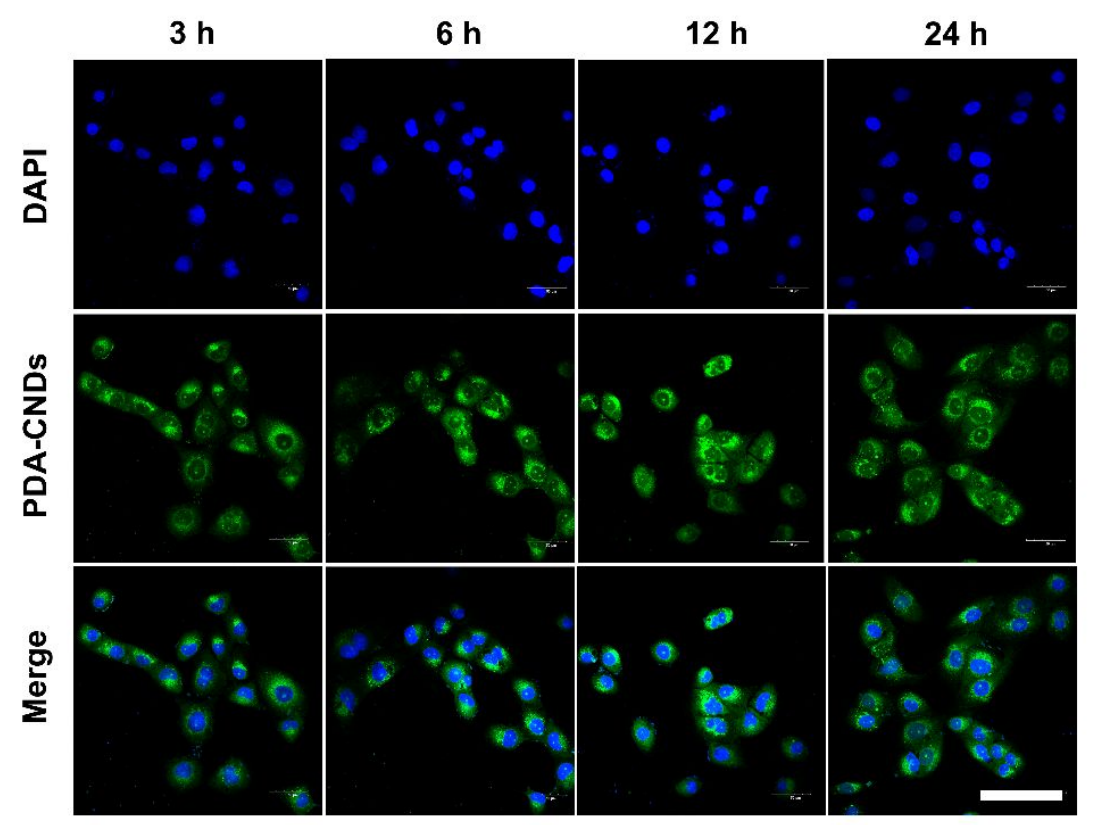

Figure S15. Representative images of PDA-CNDs uptake by HK2 cells under normoxia after PDA-CNDs incubation for different times. Nuclei were stained with DAPI. Scale bar: $100 \mu \mathrm{m}$. 


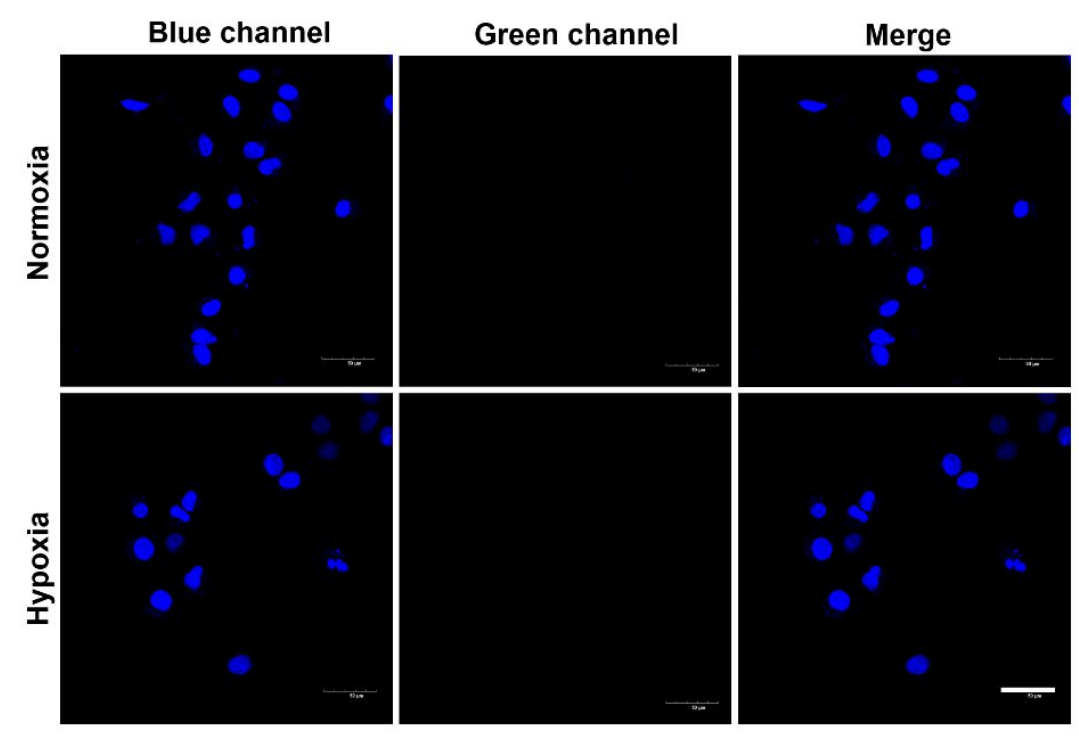

Figure S16. Representative fluorescent images of HK2 cells without PDA-CNDs treatment under both normoxia and hypoxia conditions. Scale bar: $50 \mu \mathrm{m}$.

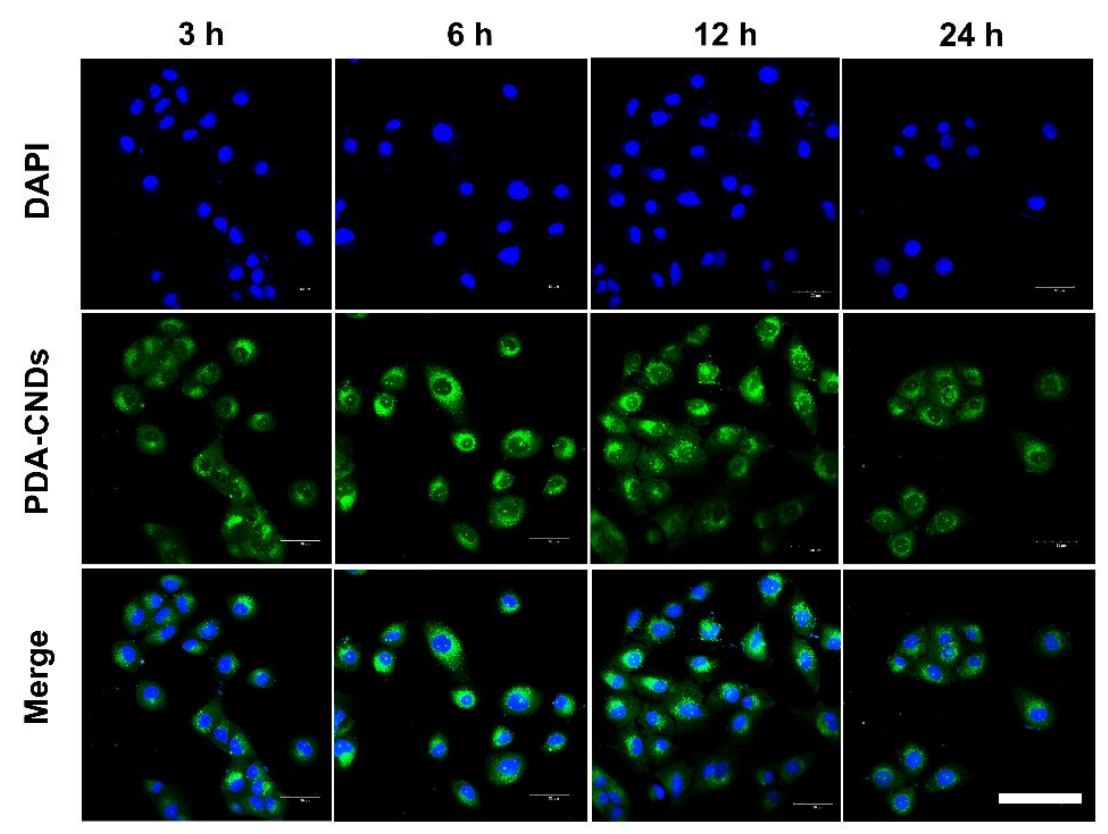

Figure S17. Representative images of PDA-CNDs uptake by HK2 cells after hypoxia $(1 \mathrm{~h})$ with PDA-CNDs incubation for different times. Nuclei were stained with DAPI. Scale bar: $100 \mu \mathrm{m}$. 


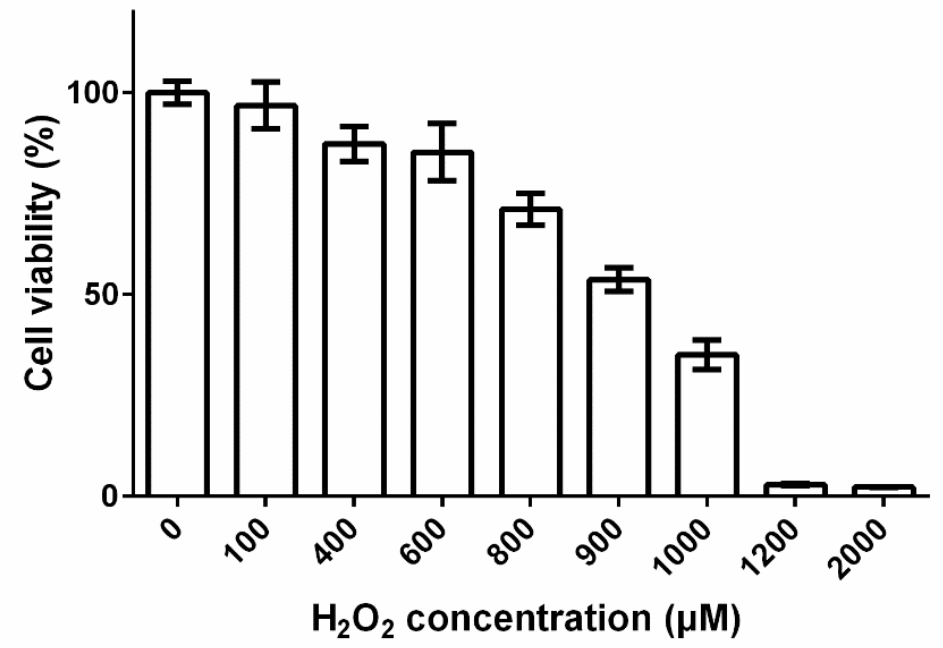

Figure S18. In vitro cell viability of HK2 cells incubated with different concentrations of $\mathrm{H}_{2} \mathrm{O}_{2}$ for $24 \mathrm{~h}$. Data are mean $\pm \mathrm{SD}$ from four independent replicates.
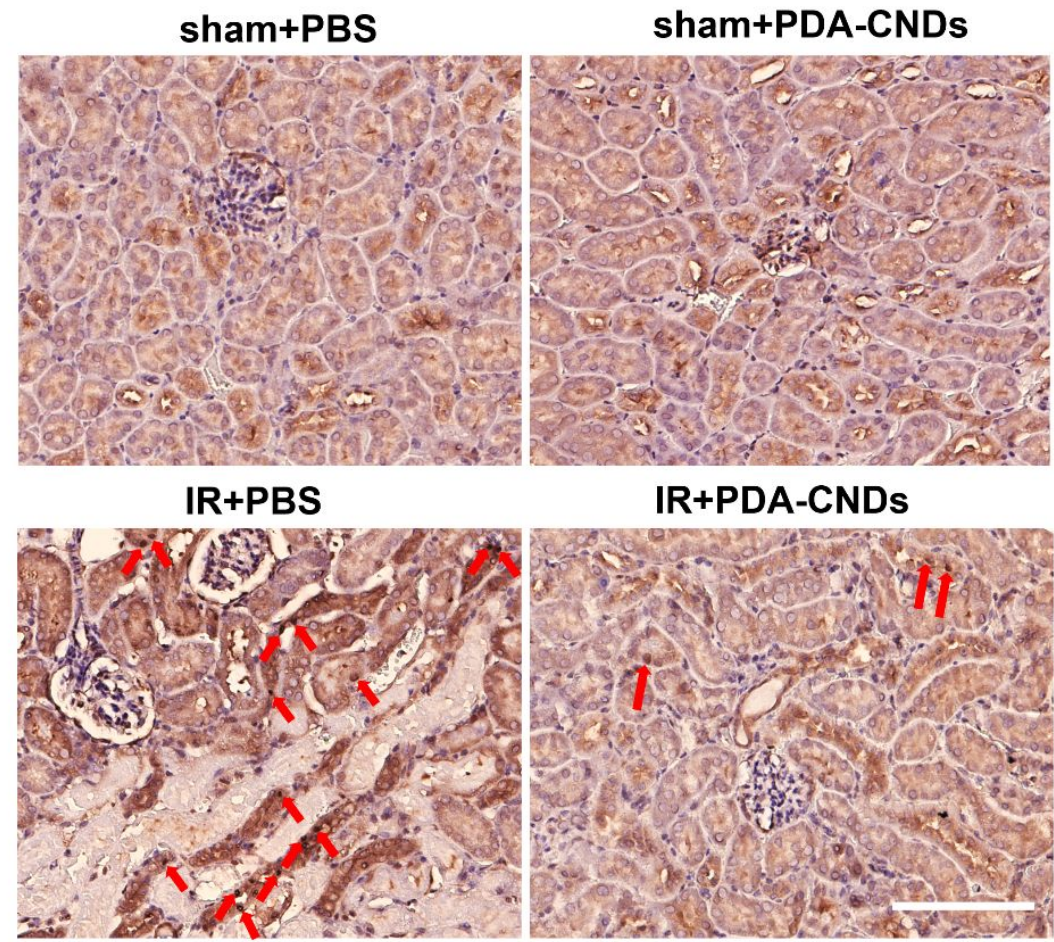

Figure S19. Representative immunohistochemical staining of p65 (NF- $\kappa B)$ in renal tissues. Nuclear p65 (NF-кB)-positive cells were indicated with red arrows. Scale bar: $100 \mu \mathrm{m}$. 

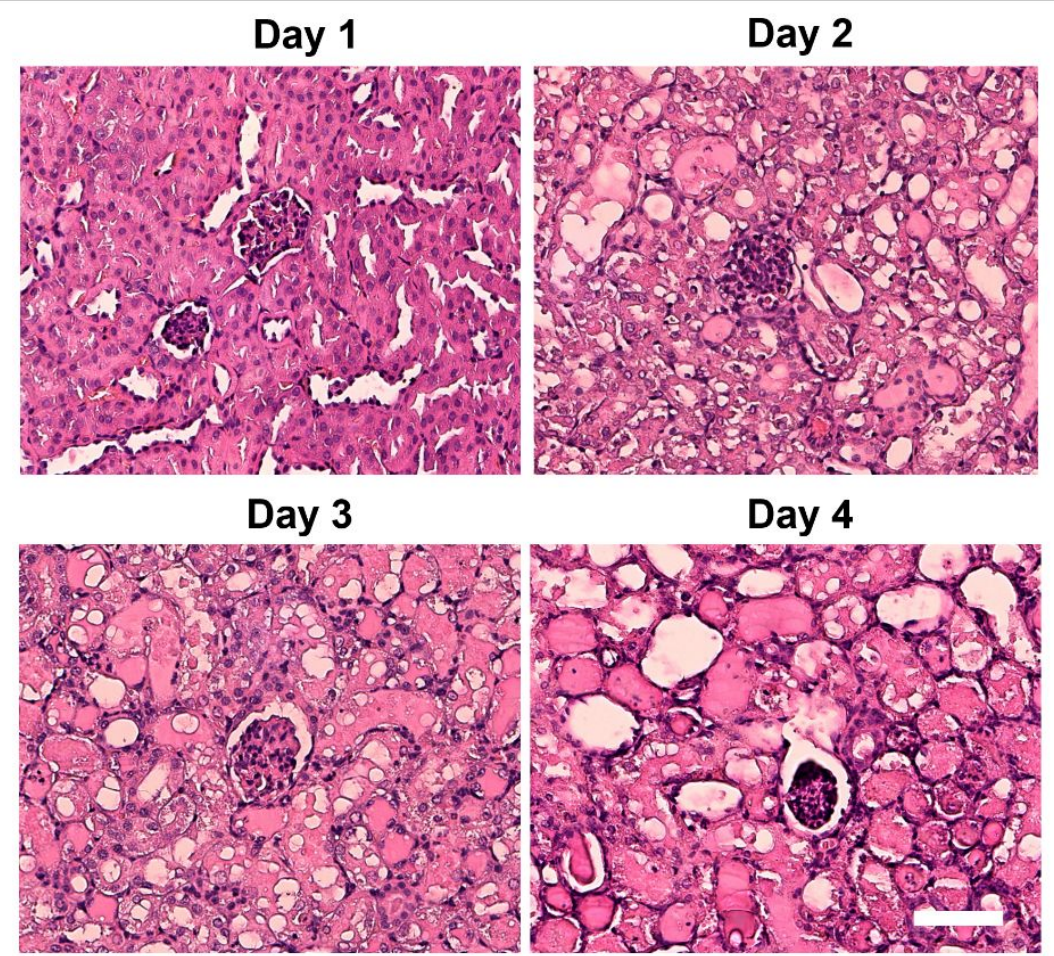

Figure S20. Representative H\&E staining of kidney sections from mice receiving cisplatin injection for different days. Scale bar: $100 \mu \mathrm{m}$.

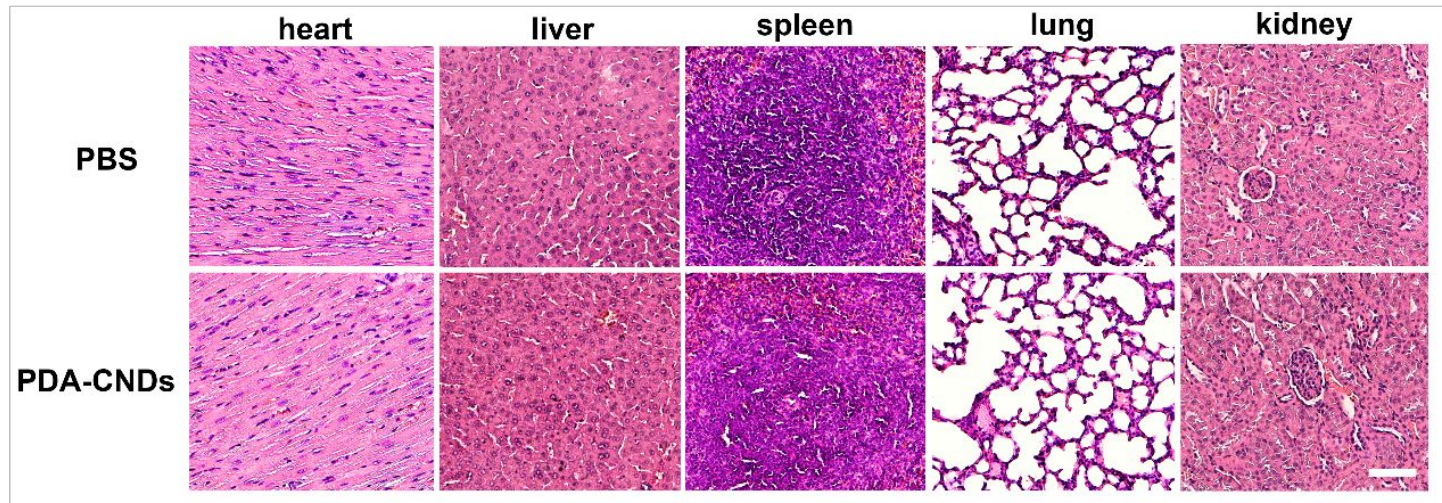

Figure S21. In vivo short-term toxicity assessment of PDA-CNDs $(24 \mathrm{~h})$.

Representative H\&E staining of major organs (heart, liver, spleen, lung and kidney). Scale bar: $100 \mu \mathrm{m}$. 




Figure S22. In vivo long-term toxicity assessment of PDA-CNDs (1 month).

Representative H\&E staining of major organs (heart, liver, spleen, lung and kidney). Scale bar: $100 \mu \mathrm{m}$. 